\title{
The Effelsberg Search for Pulsars in the Galactic Centre
}

\author{
M. Kramer, B. Klein, D. Lorimer, P. Müller, A. Jessner, R. Wielebinski \\ Max-Planck-Institut für Radioastronomie, Bonn, Germany
}

\begin{abstract}
We report the status of a search for pulsars in the Galactic Centre, using a completely revised and improved high-sensitivity doublehorn system at $4.85-\mathrm{GHz}$. We also present calculations about the success rate of periodicity searches for such a survey, showing that in contrast to conclusions in recent literature pulsars can be indeed detected at the chosen search frequency.
\end{abstract}

The detection of radio pulsars in the near vicinity of the Galactic Centre is apparently hampered by the largely increased scattering of pulsar signals at the inhomogeneities of the interstellar medium. This effect cannot be removed by instrumental means but can only be reduced by observations at high radio frequencies. The steep spectra of pulsars require a compromise in the choice of the search frequency to be used. As a consequence, we had started to search the Galactic Centre at a frequency of $4.85-\mathrm{GHz}$ using the Effelsberg $100-\mathrm{m}$ radiotelescope (Kramer et al. 1996, in Proc of IAU Colloq. 160, PASP, p. 13).

Since recently, we have employed both horns of the high-sensitive doublehom $6 \mathrm{~cm}$-frontend $\left(T_{\text {sys }} \approx 25 \mathrm{~K}\right)$ connected to four 8-channel filterbanks with $B=8 \times 80=480 \mathrm{MHz}$ bandwidth. The data are recorded by a new " $48+$ "channel backend working under VxWorks with a maximum datarate of $\sim 30$ $\mathrm{MB} / \mathrm{s}$ (10 MB/s sustained). In combination with new reduction software this results in a greatly improved sensitivity of less than $0.1 \mathrm{mJy}$ for a $12 \mathrm{~min}$ integration (Fig. 1).

Recently, Cordes \& Lazio (1997, ApJ, 475, 557, hereafter CL97) presented calculations which indicated that periodicity searches in the Galactic Centre below $10 \mathrm{GHz}$ would hardly be successful, since scattering will reduce the pulsed fraction of the pulsar signal at lower frequencies. Instead, they favoured a complementary imaging approach. It has to be noted, however, that their calculations made use of a simplified expression to describe the effect of scattering. A correct treatment shows a still significant contribution of power in the higher harmonics. As a result, the decrease of pulsed emission towards lower frequencies is much slower than derived by CL97. In fact, we demonstrate that pulsars can still be discovered in periodicity searches at $5 \mathrm{GHz}$ (Fig. 2).

Acknowledgments. We thank the receiver group of the MPIfR for building the superb $6 \mathrm{~cm}$-receiver and the corresponding filterbanks. The digital-group, in particular Thomas Kugelmeier, made significant contributions in the development of the new backend POESY. 

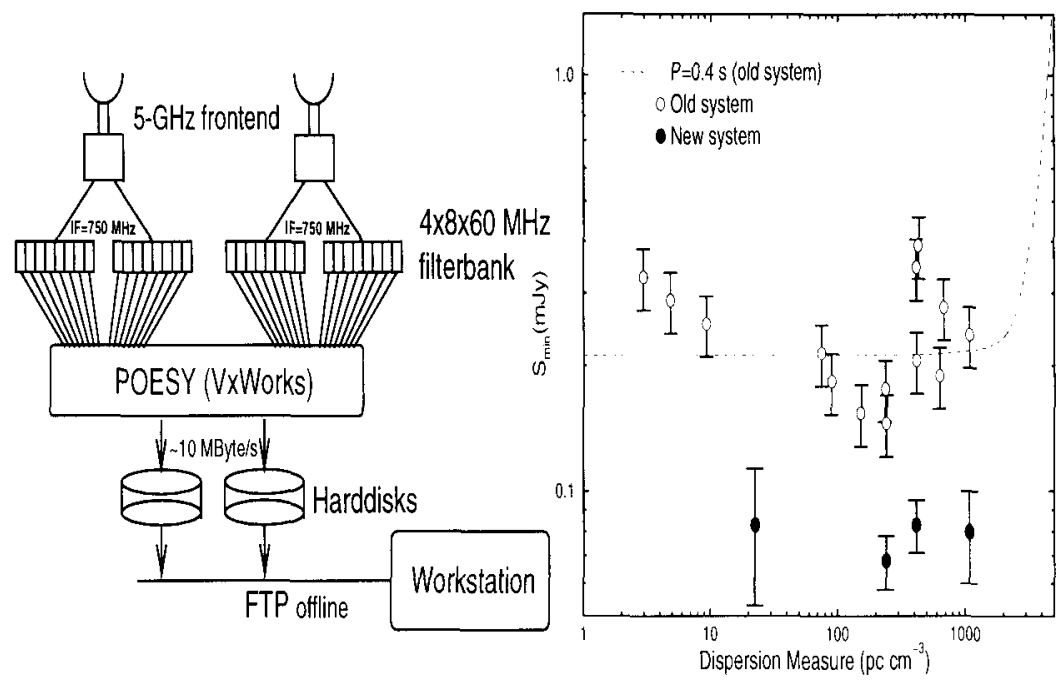

Figure 1. left) New search system for the $6 \mathrm{~cm}$ Galactic Centre survey, right) improved sensitivity of the new system in comparison to that described by Kramer et al. (1996).
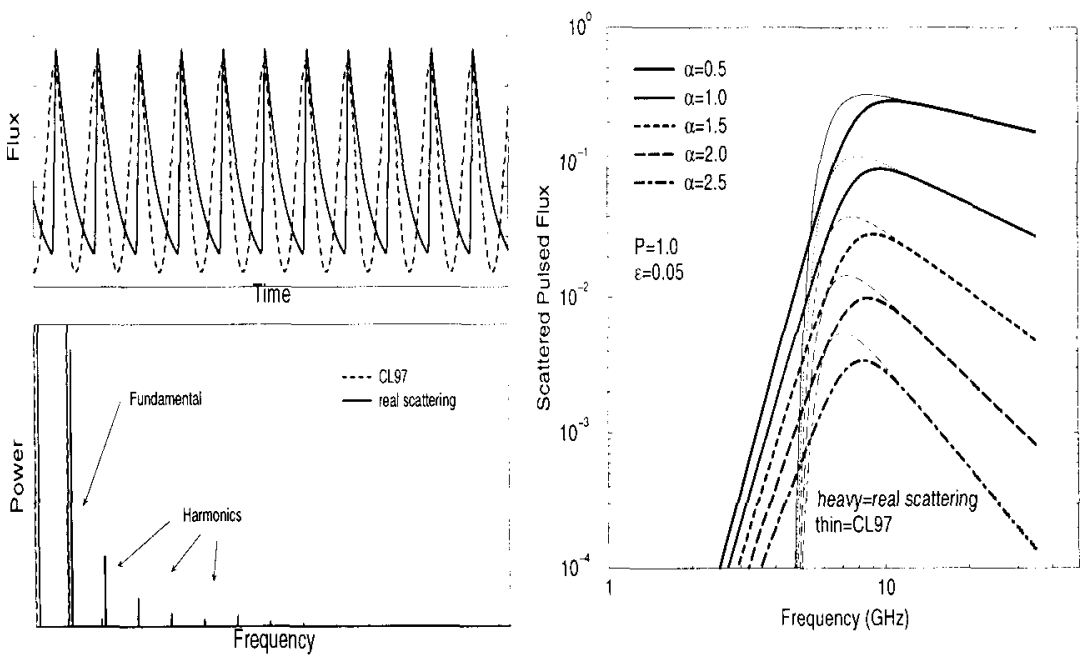

Figure 2. left) Effects of pulse scattering on time series and power spectrum when modeled as by CL97 and when applying the actual formulae, right) scattered pulsed flux for a model pulsar of $P=1 \mathrm{~s}$ and a duty cycle of $5 \%$ for different spectral indices. In contrast to results by CL97 (thin lines), real scattering (heavy lines) still produces detectable pulsed fraction at frequencies around 5 GHz. 